\title{
The influence of the study of medicine on Clément Juglar's first take on the economic cycle, 1846-1862
}

\author{
Ludovic Frobert (CNRS/Lyon), Omar Hamouda (Glendon College/Toronto)
}

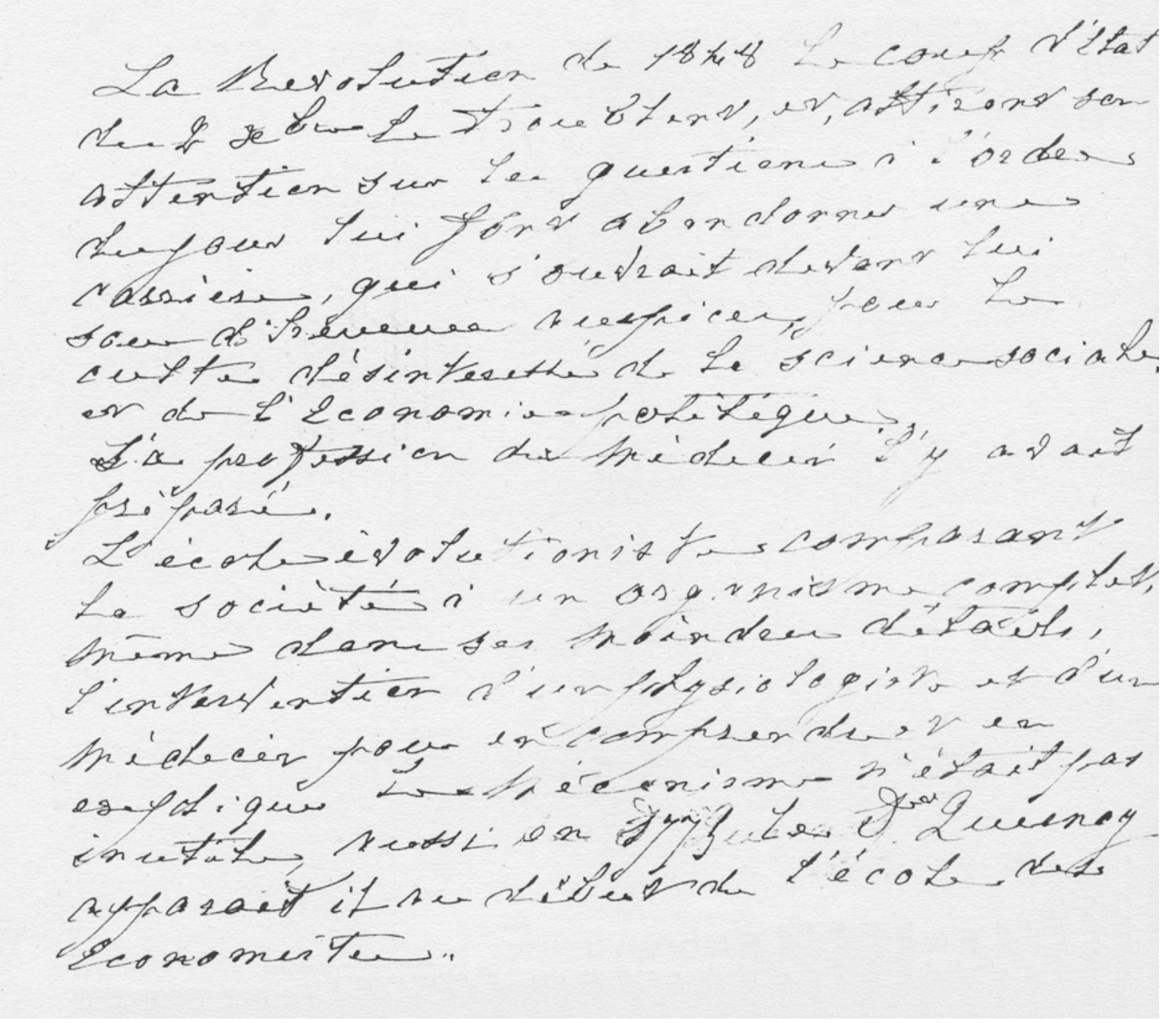

La Révolution de 1848, le coup d'Etat du 2 septembre le troublant et attirant son attention sur les questions à l'ordre du jour lui font abandonner une carrière qui s'ouvrait devant lui .... Le culte désintéressé de la science sociale et de l'économie politique.

La profession de médecin l'y avait préparé.

L'école évolutionniste comparant la société à un organisme complet même dans ses moindres détails, l'intervention d'un physiologiste et d'un médecin pour en comprendre et en expliquer le mécanisme n'était pas inutile, ainsi en 1773 le Docteur Quesnay apparaît-il au début de l'école des économistes.

[ Brouillon d'une présentation de Clément Juglar, probablement lors de sa première candidature à l'Académie des sciences morales et politiques en 1886]. 


\section{Introduction}

Dans son rapport verbal sur Des crises commerciales et de leur retour périodique en France, en Angleterre et aux Etats-Unis pendant le $19^{e}$ siècle (1862), Louis Wolowski soulignait les analogies entre «corps social» et «organisme de l'homme». Il poursuivait, que le corps social «a ses maladies, et il doit avoir son hygiène. Cette hygiène a ses règles et ses lois, et $\mathrm{M}$. le docteur Juglar a su très habilement les exposer $»^{1}$. Depuis lors, l'influence des idées médicales sur l'économie politique de Clément Juglar et notamment sur sa conception du cycle, a été de nombreuses fois questionnée $^{2}$. Récemment, alors que J. Niehans ${ }^{3}$ jugeait cette influence peu concluante et rattachait plus largement l'inspiration de Juglar au contexte historique et culturel général, P. Groenowegen ${ }^{4}$ repris par D. Besomi ${ }^{5}$ estimait, au contraire, cette influence majeure : en témoignaient chez Juglar l'identification entre maladie et crise, l'adoption d'une conception complexe de la causalité, l'influence du temps repérable aux mécanismes auto-renforçants ainsi que finalement une méthodologie où l'articulation fait/théorie était la base d'un véritable diagnostic économique et social. M. Gilman ${ }^{6}$ puis D. Pelissier ${ }^{7}$ s'appuyant sur J. Bourcier de Carbon $^{8}$, mentionnaient l'impact de la médecine expérimentale bernardienne sur Juglar.

L'objectif de ce papier est de remonter cette piste en portant attention au contexte intellectuel de l'époque. Plus précisément, nous voudrions suggérer que les thèmes médicaux ont bel et bien joué un rôle majeur dans la genèse de la « vision »" du cycle

\footnotetext{
${ }^{1}$ L. Wolowski, «Rapport verbal sur un ouvrage de M. le Docteur Clément Juglar intitulé Des crise commerciales et de leur retour périodique en France, en Angleterre et aux Etats-Unis pendant le $19^{\mathrm{e}}$ siècle», 1862.

${ }^{2}$ Voir, par exemple, à sa mort, A. De Foville, "Clément Juglar", Economic Journal, 15 (58), 1905, p. $293-298$; P. Beauregard, « Notice sur la vie et les travaux de M. Clément Juglar », Séances et travaux de l'Académie des sciences morales et politiques : Comptes-rendus, vol. 71, p. 153-179.
}

${ }^{3}$ J. Niehans, « Juglar's Credit Cycles », History of Political Economy, 24 (3), 1992, p. 547.

${ }^{4}$ P. Groenewegen, «Joseph Clément Juglar (1819-1905): From Physician to Analyst of Business Cycles”, in P. Groenewegen (ed.), Physicians and Political Economy. Six Studies of the Work of Doctor-Economists, London and NY, Routledge, 2001.

${ }^{5}$ D. Besomi, ???, ch.6, « Cl. Juglar: La Crisi come Patologia“.

${ }^{6}$ M. H. Gilman, «Clément Juglar 1819-1905, analyste des crises », in Y. Breton et M. Lutfalla (Dir.), L'Economie politique en France au 19e siècle, Paris, Economica, 1991, p. 277-302.

${ }^{7}$ D. Pellissier, « Clément Juglar : Héritage et actualité de sa théorie des cycles », in P. Dockès et alii. (Dir.), Les traditions économiques françaises 1848-1939, Paris, CNRS Editions, 2000, p. 273-286.

${ }^{8} \mathrm{~J}$. Bourcier de Carbon, Essai sur l'histoire de la pensée et des doctrines économiques, tome 2, Paris, Montchrestien, 1972, p. 73.

${ }^{9}$ Nous adoptons ici la reformulation herméneutique de la notion schumpeterienne de «vision » proposée récemment par R. Heilbronner. R. Heilbroner, «Analysis and Vision in the History of Modern Economic Thought », Journal of Economic Literature, 28, septembre 1990, p. 1097-1114; R. Heilbroner, "Economics as 
l'économie, elle donnait le nom de crise aux phénomènes morbides qui lui paraissaient se lier à l'expulsion de cette matière peccante. Ces théories surannées sont aujourd'hui abandonnées par tous les hommes de l'art, et nous n'en parlerions pas, si elles ne dominaient encore dans les idées et le langage vulgaires. Il suffit pour faire justice de ces suppositions gratuites, d'observer que c'est précisément que c'est précisément chez les personnes saines et fortes que les prétendus efforts d'élimination sont violents et incoercibles, ou, en d'autres termes, que les maladies revêtent la forme la plus aiguë, tandis qu'ils sont nuls ou presque nuls chez les individus scrofuleux, scorbutiques, etc, c'est-àdire chez ceux où il existe une altération réelle des fluides organiques. - Aujourd'hui cependant le terme de crise s'emploie encore dans le langage médical ; mais il désigne simplement la ligne de démarcation que l'on peut quelquefois entrevoir et signaler, tantôt entre l'accroissement et le décroissement d'une maladie, et tantôt entre la marche ascendante de celle-ci et la chute des forces vitales, de sorte que la crise ainsi conçue peut être interprétée comme l'expression du retour à la santé ou l'indice d'une mort prochaine. Dans les maladies, il y a en général tendance vers le retour à l'état normal, tendance qui est due à l'action des forces inhérentes à l'organisme vivant. Lorsque cette tendance est efficace, ont voit les organes dont les opérations régulières avaient été suspendues reprendre leurs fonctions caractéristiques ». que la consommation opérée durant l'exercice de la fonction, d'où nécessité de repos, intermittence, puis retour de l'activité fonctionnelle »

J. F. Bertet Dupiney de Vorepierre, Dictionnaire français illustré et encyclopédie universelle pouvant tenir lieu de tous les vocabulaires et de toutes les encyclopédies, Paris, M. Lévy frères, 1876 [ $1^{\text {ère }}$ édition 1858-1864],

«Crise », vol.1, p. 828-829

«Périodicité », vol. 2, p. 678. ${ }^{11}$

La définition des termes «Crise » et «Périodicité », renvoie directement à un paradigme médical dans lequel l'étudiant en médecine, puis le jeune Docteur Juglar a été formé à Paris, dans les années 1840. Le lieu et la période importent en histoire de la médecine. Michel Foucaut, Erwin Ackerknecht et d'autres historiens ou philosophes ont signalé que cette période vit l'Ecole clinique parisienne arriver à son apogée ${ }^{12}$. La première partie du $19^{\mathrm{e}}$ siècle enregistre la «naissance de la clinique » et c'est un véritable changement de paradigme qui s'opère. A l'ancestrale "médecine au chevet du malade » succède la «médecine hospitalière » et ce n'est qu'après 1850 que cette dernière commence à être supplantée par la «médecine de laboratoire ». Honoré de

\footnotetext{
${ }^{11}$ L'importance de ces deux entrées du Dictionnaire de Dupiney de Vorepierre avait été signalée dans P. Salmon, L'Originalité de Juglar, Thèse de la faculté de droit et sciences économiques, Université de Paris, 1966, p. 133 et suivantes. Voir également, L. N. Bescherelle, Dictionnaire national ou Dictionnaire universel de la langue française, Paris, Garnier frères, 1856 (vol. 1, p. 835 ; vol. 2, p. 845) ; M. La Châtre, Nouveau dictionnaire universel, Paris, Docks de la librairie, 1870 (vol 1.p. 1167 ; vol. 2, p. 1014).

${ }^{12}$ E. Ackerknecht, La médecine hospitalière à Paris 1794-1848, Paris, Aubier, 1986 ; M. Foucault, Naissance de la clinique, Paris, Puf, 1963.
} 
Balzac saisit au plus près cette révolution exaltant, dans La maison Nucingen, « la médecine moderne, dont le plus beau titre de gloire est d'avoir de 1799 à 1837, passé de l'état conjectural à l'état de science positive » et ajoutant que cette évolution s'était opérée sous «l'influence de la grande école analyste de Paris $»^{13}$. Dans la première moitié du $19^{\mathrm{e}}$ siècle, Paris est le principal centre de renouvellement de la médecine. Cette période voit naître l'hôpital moderne et se mettre en place les principales institutions d'enseignements, notamment les écoles de médecine. Sur le plan théorique c'est l'école anatomo-clinique qui domine alors les débats la médecine expérimentale de Claude Bernard et de Louis Pasteur ne s'imposant qu'un peu plus tard dans le siècle. Or, les «maîtres » de la Faculté de médecine de Paris à qui Juglar dédit sa thèse de 1846 sont représentatifs de la génération des « éclectiques » qui, après 1830, cherchent à dépasser les controverses antérieures entre le physiologisme de François Broussais et le clinicisme de Jean Nicolas Corvisart, Gaspard Laurent Bayle ou René Théophile Laennec. Cette génération, moins doctrinale et moins systématique, coïncide avec la période la plus brillante de la médecine hospitalière parisienne. Parmi les maîtres de Juglar, Auguste-François Chomel (1788-1856) est certainement le plus connu et le plus représentatif ; ce spécialiste d'anatomie pathologique, féru de la méthode numérique (voir plus loin) était le successeur de Laennec à l'hôpital de la Charité (1827), probablement le clinicien le plus éminent de Paris au tournant des années 1840 et l'un des grands professeurs de la faculté de médecine de Paris. Les autres noms cités par Juglar sont tous des chirurgiens ou des spécialistes de l'anatomie pathologique ${ }^{14}$ : ces médecins suivaient le retournement opéré précédemment par François-Xavier Bichat qui avait enseigné que la mort était le miroir de la vie et ils étaient ensemble partisans d'une médecine des lésions qui s'était substituée à une médecine des symptômes et qui se caractérisait par quelques traits dominants, empirisme, spécialisation, scepticisme thérapeutique. Chomel définit d'ailleurs de la façon suivante le prosaïsme de la « doctrine de l'Ecole de Paris »:

Cette doctrine se distingue des autres, non par une de ces théories quelquefois brillantes et toujours erronées, qui prétendent expliquer, à l'aide d'une hypothèse, tous les phénomènes de la vie, mais par une tendance constante et une impulsion active vers ce qu'il y a de positif en médecine, c'est-à-dire les faits bien observés et les conséquences rigoureuses qui en découlent ${ }^{15}$.

Ce qui importait pour ces médecins de l'âge positif était le repérage des lésions inférées de l'étude rigoureuse par la vue, l'ouie, le toucher (ces sens eux-mêmes relayés par les nouvelles techniques de l'auscultation médiate, comme le stéthoscope mis au point par Laennec à l'Hôpital Necker en 1816) des différents signes émis par le corps. Juglar, étudiant au début des années 1840 a été formé par cette grande génération éclectique : dans ses cahiers de médecine on retrouve les notes abondantes qu'il a prise, dans les années 1841-1844 sur les grandes références de cette tradition : sur le Traité de

\footnotetext{
${ }^{13}$ M. Grmek, «Le concept de maladie », in M. Grmek (dir.), Histoire de la pensée médicale en Occident, vol. 2 , Paris, Seuil, 1999, p. 147.

${ }^{14}$ Les autres «maîtres » salués par Juglar dans sa thèse étaient : Auguste Bérard (chirurgien), membre de l'Académie Royale de Médecine, fondateur de la Société de chirurgie de Paris ; il avait la chaire de clinique chirurgicale à la faculté de médecine. Léon Auvity était chirurgien à l'hôpital des Enfans-Trouvés et chirurgien-major de la garde municipale de Paris. Jacques Baron travaillait aussi aux Enfans-Trouvés, membre de l'Académie Royale de Médecine (section d'anatomie pathologique, comme F.Chomel). E. R. A Serres, anatomiste, membre de l'Académie Royale de Médecine (section anatomie et physiologie). Détails biographiques dans C. Lachaise, Les médecins de Paris jugés par leurs œuvres ou Statistique scientifique et morale des médecins de Paris, Paris, Sachaile, 1845.
}

${ }^{15}$ F. Chomel, Elements de pathologie générale, Paris, Masson, $4^{\mathrm{e}}$ édition [ $1^{\mathrm{er}}$ édition 1817] 1856, p. VII. 
Thérapeutique d'Armand Trousseau ou sur le cours de pathologie générale de Chomel qui s'ouvre par la définition suivante de la maladie : « un désordre notable survenu soit dans la disposition matérielle des parties constituantes du corps vivant soit dans l'exercice de ses fonctions $»^{16}$. Significativement, sa Thèse de doctorat en médecine consacrée à l'influence des maladies du cœur sur les poumons porte en exergue une citation tirée des Recherches sur la vie et la mort de François-Xavier Bichat. Ce travail de Thèse est caractéristique d'une étape avancée de l'Ecole Clinique parisienne puisque Juglar se propose d'étudier la combinaison d'effets que peut avoir un organe « mécanique » comme le cour et un organe surtout « chimique » comme le poumon sur «l'accomplissement régulier de la vie». Pour cela il s'appuie, là encore, sur des titres caractéristiques notamment, la Clinique médicale de Gabriel Andral, le Traité d'anatomie pathologique générale de Jean Cruveilhier ou les travaux publiés par Pierre Louis dans les Mémoires de la société médicale d'observation. Et dès l'introduction, il explique que pour démontrer « il faut prouver et voir si l'anatomie pathologique, les symptômes, sont en rapport $\gg^{17}$ avec les thèses avancées.

\section{La méthodologie de Juglar : empirisme et statistiques}

Sur le plan méthodologique, Juglar est un empiriste. Son empirisme s'appuie sur l'usage intensif et qualitatif des statistiques ${ }^{18}$. Dès son premiers travail sur le cycle (1856) il utilisent les séries longues d'un grand nombre d'indicateurs, dont notamment, les variations de l'escompte, des importation et exportation, des données démographiques, du prix des céréales. L'année suivante dans les articles du Journal des économistes les données sont encore plus importantes.

Ces séries longues sont classées, étudiées et comparées dans le cadre de tableaux synoptiques. Ces tableaux permettent à Juglar de repérer une série témoin, celle des variations du taux de l'escompte. Il explique ainsi que cette série constitue le « point de comparaison » [1857a:37] de l'ensemble général des fluctuations, celui qui « en donne le tableau le plus fidèle » [1857b : 255]. Mais pour quelle raison ? Pour des raisons essentiellement quantitative, Juglar expliquant que c'est cette série qui présente « la plus grande régularité et les différences les plus sensibles» [1856: 559]. A partir des variations significatives de l'escompte et du mouvement des encaisses ont peut dès lors suivre les variations de toutes les autres données économiques et démographiques qui elles présentent aussi des «différences maxima et minima» marquées mais toutefois moins « sensibles » [1857b 255] que pour les escomptes. L'étude individuelle de chaque cycle confirme également ce point. Chaque cas précis, avec ses propres singularités historiques (politique notamment), permet de repérer les mêmes variations quantitatives.

Quelles influences sont repérables dans l'usage que fait Juglar des statistiques? Il est d'abord crucial de rappeler que les années 1830-1850 définissent traditionnellement, selon l'expression de H. Westergaard, "l'ère de l'enthousiasme statistique ». En France c'est en 1833 qu'est créée la Statistique Générale de la France, placée initialement sous

\footnotetext{
${ }^{16} \mathrm{Cl}$. Juglar, Cahiers de médecine, archives familiales.

${ }^{17}$ C. Juglar, De l'influence des maladies du coeur sur les poumons, Paris, Masson, 1846, p. 7-8.

${ }^{18}$ M. Morgan, The Development of Econometric Ideas, Cambridge University Press, 1990, p. 41-44.
} 
l'autorité du Ministère de Commerce ${ }^{19}$. Collectant et organisant en tableaux les statistiques des autres administrations, ce service dirigé d'abord par Alexandre Moreau de Jonnès puis à partir de 1852 par Alfred Legoyt (qui fournira à Juglar une partie de sa documentation), organise et exploite les recensements quinquennaux et analyse le mouvement de la population. Cet essor institutionnel de la statistique n'est pas sans influence sur le groupe des économistes libéraux français qu'a rejoint Juglar des le tournant des années $1850^{20}$. Prenant leurs distances vis-à-vis des critiques adressés par J.-B. Say à l'usage des statistiques, ils en soulignent désormais la fécondité. Louis Wolowski dans ses Etudes d'économie politique et de statistique (1848), dédiées à Adolphe Quételet, écrit par exemple :

La statistique fait connaître la marche et le développement des phénomènes sociaux, au moyen d'états numériques, qui constatent la situation successive de la société sous les divers aspects, dont la connaissance est nécessaire pour apprécier les éléments de l'existence politique, civile, intellectuelle, morale et industrielle des nations ${ }^{21}$.

Et, peu de temps après, l'article «Statistiques» du Dictionnaire de l'économie politique (signé par Joseph Garnier) confirme cette alliance nouvelle entre statistique et économie politique libérale ${ }^{22}$. Ce sont également les hygiénistes qui promeuvent de leur côté le développement des statistiques. A partir de 1829, ces hygiénistes - parmi lesquels Louis-René Villermé, Alexandre Parent-Dûchatelet, Louis Benoiston de Châteauneuf, etc - ont créé les Annales d'hygiène publique et de médecine légale où, en particulier jusqu'à 1853, vont être franchis deux caps théoriques décisifs : d'une part l'usage systématique de la méthode numérique pour mesurer précisément l'influence sur la santé de telle ou telle population des conditions d'environnement spécifiques ; d'autre part la mise en retrait des facteurs d'environnement naturel progressivement remplacés par les facteurs techniques et surtout sociaux ${ }^{23}$.

L'influence de ces différents milieux - les administrateurs de la statistique, les économistes libéraux, les hygiénistes - sur Juglar est incontestable. Et d'autres sources probables pourraient encore être mentionnées, notamment l'influence des feuilles financières et boursières très friandes de statistiques et en pleine expansion à partir de 1836 (voir plus loin). Mais une autre influence, lui venant directement de l'école anatomo-clinique, et sans doute plus importante encore. C'est principalement en

\footnotetext{
19 A. Desrosières, La politique des grands nombres: une histoire de la raison statistique, Paris. Voir également, H. Le Bras, « La Statistique Générale de la France » in P. Nora (dir.), Les lieux de mémoire, tome 2, Paris, Gallimard, 1986, p. 340-344 ; T. Porter, The Rise of Statistical Thinking 1820-1900, Princeton University Press, 1986, p. 27-30.

${ }^{20}$ Juglar intègre la Société d'économie politique en 1852. Ses premiers travaux économiques et démographiques sont publiés entre 1851 et 1854 dans le Journal des économistes, organe de l'Ecole libérale de Paris.

${ }^{21}$ L. Wolowski, Etudes d'économie politique et de statistique, Paris, Guillaumin, 1848, p. 418.

${ }^{22}$ J. Garnier, «Statistiques », in C. Coquelin et Guillaumin, Dictionnaire de l'économie politique, Paris, Guillaumin, 1852, p.

${ }^{23}$ B. P. Lécuyer, « Médecins et observateurs sociaux : les Annales d'hygiène publique et de médecine légale (1820-1850) », in, Pour une histoire de la statistique, Paris, Economica, 1987, p. 445-475. J. Léonard, La médecine entre les savoirs et les pouvoirs, Paris, Aubier, 1981, ch. IX.
} 
médecine que le débat sur l'utilité des statistiques fit rage dans les années $1830^{24}$. Le débat avait été lancé en 1828 par Pierre Louis qui s'appuyant sur la "méthode numérique » avait fortement nuancé les conclusions de Broussais sur les vertus thérapeutiques de la saignée. Attaqué en particulier sur sa méthode, Louis avait répliqué en 1835 consacrant un chapitre de son travail à souligner que «l'expérience véritable en médecine, ne peut résulter que de l'analyse exacte de faits nombreux, bien constatés, classés d'après leur ressemblance, comparés avec soin, et comptés $»^{25}$. Lors des très vives controverses qui se développèrent au milieu des années 1830, à l'Académie des sciences (1835) puis à l'Académie Royale de Médecine (1837) Louis et ses disciples qui avaient créés en 1832 la Société médicale d'observation (SMO) - allaient recevoir le soutien de grands cliniciens comme Jean-Baptiste Bouillaud, Gabriel Andral ou surtout Chomel, ce dernier ayant protégé et laissé travaillé Louis dans son service à l'Hôpital de la Charité. Chomel, l'un des deux présidents d'honneur de la SMO, fera d'ailleurs l'éloge de l'analyse numérique dans ses Eléments de pathologie générale définissant cette approche de la façon suivante :

Compter les faits après les avoir recueillis, chercher à déduire de leur rapprochement et de leur nombre quelques résultats exacts, soit sur les causes qui ont préparé ou déterminé la maladie, soit sur les phénomènes qui la caractérisent, sur son cours et sur ses terminaisons variées, soit enfin sur l'influence des moyens de traitement qu'on lui a opposés ${ }^{26}$.

L'essor de la méthode numérique résultait des limites de l'approche anatomo-clinique telle que l'avaient développés les pionniers, de Bayle à Laennec: les maladie étaient identifiées et classées mais les causes demeuraient hors d'atteinte et les thérapies étaient découvertes le plus souvent par hasard. Dans ce contexte, à partir de 1830 les numéristes allaient doter cet empirisme d'une véritable méthode. Plusieurs caractéristiques du numérisme sont spécifiques et signalent une nette parenté par rapport à l'approche que développera quelques années plus tard Juglar en économie politique. D'abord, l'utilisation des statistiques diffère ici radicalement de celle des hygiénistes. Ces derniers proposaient une doctrine médicale basée sur les recherches statistiques mais à l'usage des administrateurs et des gouvernants. Les numéristes, en revanche, appliquent la recherche statistique directement à la maladie et le nombre permet ici de progresser sur les chapitres de la symptomatologie, de la thérapeutique et de l'étiologie de chaque affection particulière. Ensuite, pour Louis, la statistique n'est pas une simple annexe méthodologique mais devient une des modalités obligées de toute recherche médicale et l'un des principes décisifs de toute analyse et de toute preuve. La statistique vérifie une des conditions majeures de l'observation rigoureuse, telle que la réclame l'analyse et la pratique clinique : fournir des faits identiquement sûrs, complets, précis. Enfin, les numéristes s'obligent à l'analyse complète de chaque cas en utilisant des tableaux lisibles selon deux directions : en ligne où ils fournissent la chronologie de l'affection et en colonne où sont dissociées et réparties les différentes données sémiologiques et thérapeutiques; le clinicien peut alors repérer des «lois» par la

${ }^{24}$ P. Armitage, «Trials and Errors: The Emergence of Clinical Statistics », Journal of the Royal Statistical Society. Series A, 146 (4), 1983, p. 323-326. Et surtout, J. Piquemal, « Succès et décadence de la méthode numérique en France à l'époque de Pierre-Charles-Alexandre Louis », in J. Piquemal, Essais et leçons d'histoire de la médecine moderne et de la biologie, Paris, PUF, 1993.

${ }^{25}$ P. Louis, Recherches sur les effets de la saignée dans quelques maladies inflammatoires, Paris, Baillière, 1835, p. 98.

${ }^{26}$ F. Chomel, Eléments de pathologie générale, Paris, Masson, $4^{\text {e }}$ ed., 1856, p. 589. 
présence ou l'absence constantes de certains signes et lésions, par la fréquence de telle ou telle association, etc.

Juglar lui-même s'est inscrit, lors de son travail de Thèse en médecine, dans cet environnement numériste. Dans l'introduction il explique qu'il s'est dégagé des «solutions hypothétiques » au problème posé, qu'il lui a manqué « une pratique beaucoup plus longue que celle qu'il [a] pu acquérir dans les hôpitaux » et qu'il s'est appuyé donc sur la littérature positive sur le sujet ; il ajoute,

j'ai extrait des auteurs les observations qui venaient corroborer le petit nombre de faits que j'avais observé, je les ai réunis et groupés, en m'efforçant de faire voir les liens par lesquels les accidents s'unissent, s'enchaînent, l'ordre dans lequel ils se succèdent ${ }^{27}$.

Il est ainsi probable que la méthodologie empiriste de Juglar appuyée sur l'usage systématique et intensif des statistiques, a largement emprunté aux idées des « maîtres » cliniciens qu'il avait eu à la faculté de médecine de Paris dans la première moitié des années 1840 ; à Chomel, par exemple, qui refusait les systèmes et estimait oiseux et dangereux d'enquêter sur les causes des maladies ou sur les effets des remèdes et qui protégeait les numéristes comme Louis qui, comme l'a noté opportunément Ackerknecht, « fut le premier à vouloir faire d'elles [les statistiques] le fondement de la médecine $»^{28}$. Sur ce point d'ailleurs, il faut encore écouter Jules Gavarret qui, évoquant cette conjoncture scientifique en médecine notait, en 1840 :

A l'époque où nous commençâmes nos études médicales, on n'entendait parler partout que de propositions mathématiquement prouvées, de lois mathématiquement établies. Depuis le professeur et l'Académicien, jusqu'au plus humble élève, tout le monde tenait le même langage ${ }^{29}$.

\section{Le cycle comme phénomène normal}

Il y a, dès les premiers textes de Juglar, une analyse du cycle économique qui sollicite faits et théorie. Des séries statistiques sont mobilisées pour étayer une théorie faisant des variations de l'escompte la variable la plus importante du cycle. Deux caractères originaux de cette analyse sont le plus souvent mentionnés par les commentateurs : premièrement le fait de proposer une approche endogène du cycle, deuxièmement, et corrélativement, le fait que chez Juglar la seule cause de la dépression est la prospérité qui la précède ${ }^{30}$. Mais il est indispensable d'apercevoir qu'en deçà de cette analyse Juglar appuie toute son approche sur trois idées capitales :

\footnotetext{
${ }^{27}$ Cl. Juglar, De l'influence des maladies du cœur sur les poumons, ouv. cit., p. 10.

${ }^{28}$ E. Ackerknecht, La médecine hospitalière à Paris (1794-1848), ouv. cit., p. 25.

${ }^{29}$ J. Gavarret, Principes généraux de statistiques médicales, 1840, cité in J. Piquemal, art. cit., p. 81-82.

${ }^{30}$ Voir la présentation récente de M. Dal-Pont et H. Hagemann, « Business Cycles in Juglar and Schumpeter », paper presented ESHET 2005, University of Stirling.
} 
- Le cycle est organiquement lié à la constitution nouvelle des grandes nations industrielles. C'est un phénomène nouveau propre à la constitution originale des nouvelles économies dans lesquelles l'ancienne base rurale est (très) progressivement entamée par les nouvelles activités industrielles. Le temps économique évolue et à la longue durée des changements agricoles, à l'inertie des situations et la reproduction des routines, succèdent des évolutions beaucoup plus rapides et, en quelques sorte, une accélération du temps économique. Si le phénomène du cycle est désormais récurrent et ne peut être éliminé c'est en raison de deux caractères économiques nouveaux qu'identifie immédiatement Juglar : le développement de la grande industrie et des grandes entreprises, d'une part, celui de la spéculation d'autre part. Juglar prévoit l'allongement du processus de production, la longueur des délais, l'extension géographique des marchés et montre alors que le nouveau système, dominé par l'incertitude et par l'extrême difficulté à coordonner les activités, est donc sujet à des phénomènes collectifs d'emballement. Il souligne ainsi comment en phase de hausse les principaux acteurs placent « une confiance sans borne dans l'avenir, qu'on se représente sous les couleurs les plus brillantes » et comment, dans ces conditions, « la passion du jeu s'empare de toutes les imaginations » (1857:57 et 59). En bref, « ces excès de la spéculation sont trop dans la nature humaine pour qu'on puisse les prévenir par aucune mesure » (1857: 37). Face à ces caractères nouveaux de la production industrielle, la banque ne peut que suivre le mouvement.

- Si l'on parle désormais de «nations industrielles » c'est aussi pour souligner la détermination économique de tous les autres phénomènes sociaux et politiques. Juglar le remarque : « au lieu d'attribuer le malaise commercial aux troubles et aux révolutions, il faudrait chercher la cause et l'origine de ces dernières dans les écarts de la spéculation et l'encombrement des fabriques » (1857 : 38). Il n'est donc pas étonnant que les fluctuations affectent l'ensemble des phénomènes économiques (commerciaux et bancaires) mais également les principaux mouvements de la population (naissances, décès, mariages). Juglar s'inscrit ici, en la prolongeant dans la tradition industrialiste et libérale qui prévoit la substitution des anciennes sociétés guerrières, belliqueuses, violentes, sujettes aux révoltes, par les nouvelles sociétés industrielle, pacifiques et progressives.

- $\quad$ Peut-on combattre ces phénomènes et est-il seulement souhaitable de le faire ? Pour Juglar le cycle est un véritable rythme de croissance du système nouveau : «Plusieurs fois, depuis le commencement de ce siècle, nous avons passé par de semblables gênes, pour en sortir plus vigoureux que jamais et atteindre un développement commercial inespéré » (1857: 36). Ce rythme est la contrepartie du caractère innovatif de l'industrie moderne et sa phase décroissante sanctionne les faibles et les imprudents. En revanche, « les autres, assez robustes pour résister, reprennent le cours de leurs opérations avec une vigueur nouvelle, débarrassés des obstacles d'une imprudente spéculation» (1857: 38-39). Significativement, Juglar contraste la situation de l'art et du commerce. Dans l'art également, en tous lieux et tous temps, on observe des «époques de renaissance et de décadence»; mais une grande différence s'observe : «si, par un procédé graphique usité en statistique, on veut les représenter par les ondulations d'une ligne, on observe que les sommets de l'art vont s'abaissant toujours, 
tandis que ceux du commerce et de l'industrie s'élèvent sans cesse dans des proportions telles qu'aujourd'hui le monde entier leur sert de base » (1862 : VII).

Où Juglar a-t-il pu puiser ou renforcer cette conception de la normalité du cycle ? Un premier point est à souligner: l'idée de cycle continue à demeurer absente de la pensée libérale de son temps. Ceci se vérifie aussi bien dans la série d'articles publiés dans le Journal des économistes au moment de la crise économique de $1847^{31}$ que dans l'entrée «Crise» du Dictionnaire de l'économie politique (1852). Dans ce dernier travail, Charles Coquelin définit la crise comme un « dérangement subit des affaires, qui en trouble la marche et dans une certains mesure en suspend le cours $»^{32}$. La crise auparavant avait une origine exclusivement exogène mais avec le développement récent du crédit elle est devenue " presque $^{33}$ périodique ». Ce caractère nouveau s'explique toutefois par un facteur institutionnel, la «mauvaise constitution du crédit» et, plus précisément, «le monopole exercé par certaines branches privilégiées $»^{34}$; mais ces crises « cesseront de se produire $»^{35}$ avec la pleine liberté des banques. On vérifie ici que chez les libéraux proches de Juglar il y a absence complète de l'idée de cycle, conçu comme phénomène normal, et réitération de l'ancienne idée de l'impossibilité d'une surproduction générale, des accidents ponctuels pouvant toujours se produire mais pour des raisons essentiellement exogènes, politiques ou naturelles. En revanche, l'idée de cycle est bien présente dans d'autres secteurs de l'opinion au moment où Juglar va publier ses premiers textes ; la périodicité du phénomène a été rapidement repérée par la presse financière. Dans la seconde moitié des années 1830, en pleine de libéralisation de la presse en France et au moment où se développe la presse à bon marché, sont créés L'Actionnaire, L'Europe Industrielle, La Bourse, L'Egide et d'autres feuilles encore ${ }^{36}$. Représentatives des milieux d'affaire (souvent protectionnistes et interventionnistes) elles identifient rapidement, quoique de façon assez artisanale, la présence du cycle. En décembre 1839 un journaliste de L'Actionnaire explique, « on a pu remarquer que les crises se renouvellent avec une sorte de périodicité en sorte que les hommes de prévision on pu en annoncer le retour plusieurs années d'avance et à des époques à peu près déterminées ${ }^{37}$. L'idée de cycle est aussi présente, et centrale, dans la pensée socialiste. La succession de périodes critiques et organiques rythment le développement aussi bien pour les

\footnotetext{
${ }^{31}$ G. de Molinari, «La crise financière et commerciale en Angleterre », Journal des économistes, juin 1847, p. 274-284 ; H. Say, «La crise financière et la banque de France », Journal des économistes, février 1847, p. 193207 ; « La crise commerciale », Journal des économistes, 1848; « La crise financière et commerciale », Journal des économistes, avril 1848, p. 15-24; L. Faucher, « De la crise financière dans la Grande-Bretagne », Journal des économistes, novembre 1847, p. 313-328.

${ }^{32}$ C. Coquelin, «Crises commerciales », in C. Coquelin et Guillaumin, Dictionnaire de l'économie politique, Paris, Guillaumin, 1852, p. 526.

${ }^{33}$ C'est nous qui soulignons.

${ }^{34}$ Ibid., p. 530.

${ }^{35}$ Ibid., p. 534.

${ }^{36}$ Voir ici, B. Gilles, «Les crises vues par la presse économique et financière (1815-1848) », Revue d'histoire moderne et contemporaine, vol. 11, janvier-mars 1964

${ }^{37}$ Cité dans B. Gilles, p.
} 
saint-simoniens que pour les fouriéristes ${ }^{38}$; E. Buret prolonge dans De la misère des classes ouvrières en Angleterre (1840), les efforts de Sismondi pour identifier les pathologies du capitalisme et considère la récurrence des crises comme le principal indice des dysfonctionnements du capitalisme. Et, au début des années 1840 le thème du cycle se dessine aussi bien chez Proudhon ${ }^{39}$ que chez Engels et Marx. Ce dernier, autour de 1848 explique qu'on ne peut, en matière de lois économiques propres au capitalisme limiter l'observation à une seule année. En effet, « il faut toujours prendre le terme moyen de six ou sept ans - laps de temps pendant lequel l'industrie moderne passe par les différentes phases de prospérité, de surproduction, de stagnation, de crise et achève son cycle fatal $\gg^{40}$. Dans la pensée socialiste de 1848 le cycle constitue l'indice du caractère «critique » de la période capitaliste et annonce et précipite son prochain remplacement par un système plus « organique ».

Le thème de la normalité du cycle chez Juglar a pu emprunter à ses différents secteurs - dirigiste/protectionniste et socialiste - de l'opinion de son temps. Il est en particulier indéniable que, grand lecteur de la presse financière, il n'a pu qu'être attentif aux nombreux articles mentionnant les «crises commerciales » et leur périodicité mesurée à 6/7 ans grâce à différents «thermomètres » (chiffrant, en particulier par l'utilisation de séries continues, les mouvements des caisses d'épargne). Mais là encore on peut repérer chez Juglar l'influence de la médecine de son temps et notamment celle de l'Ecole clinique parisienne. Au cours de la première moitié du $19^{\mathrm{e}}$ siècle la notion hippocratique de crise est dévaluée, elle est, pour reprendre les termes de 1849, «tombée de nos jours dans un immense discrédit $»^{41}$. Lui succède une définition profondément renouvelée de la maladie : c'est une définition ontologique de la maladie qui est désormais définitivement écartée. La maladie n'est plus perçue comme une unité indépendante, une entité étrangère venant troubler le corps et ses fonctions, mais comme soit un dérèglement de fonction, soit l'indice d'une lésion organique. Développant l'intuition novatrice de Bichat qui avait expliqué que « la vie est l'ensemble des fonctions qui résistent à la mort » ces cliniciens parisiens - mais aussi les premiers tenants de la médecine expérimentale, notamment Magendie dont Juglar avait suivi le cours au Collège de France - avaient affirmé l'identité fondamentale entre processus physiologiques et processus pathologiques. Seule une différence quantitative permettait de les séparer et, dans cet ensemble, les états pathologiques n'étaient que des simples déviations qui permettaient de mieux étudier les états normaux ${ }^{42}$. Chomel exprime le plus simplement cette idée en avouant, «la pathologie n'est-elle pas, à proprement parler, la médecine elle-même ? ${ }^{43}$. Or, la solidarité entre le pathologique et le normal est également au cœur de la vision du cycle de Juglar ; et c'est probablement sur ce point précis que l'emprunt à la médecine de son

\footnotetext{
${ }^{38}$ A. Le Bras-Chopard, «Philosophies socialistes de l'histoire avant Marx » et P. Régnier, «Les socialismes originels : de Saint-Simon à Proudhon », in I. Poutrin (Dir.), Le $19^{\mathrm{e}}$ siècle : Science, politique et tradition, Paris, Berger-Levrault, 1995.

${ }^{39}$ G. Marcy, «La théorie des crises chez les socialistes français au $19^{\mathrm{e}}$ siècle », in Mélanges économiques dédiés à M. le Professeur Gonnard, Paris, LGDJ, 1946.

${ }^{40}$ K. Marx, « Discours sur le libre-échange » [1848], in Euvres économiques....

${ }^{41}$ Beaude, Dictionnaire de médecine usuelle, tome I, Paris, 1849, p. 465.

${ }^{42}$ Voir ici, G. Canguilhem, Le normal et le pathologique, Paris, Puf, 1966 (ch.II).

${ }^{43}$ A. F. Chomel, ouv. cit., p. 2.
} 
temps a été, sur le plan cognitif, le plus fécond. La conception exogène et ponctuelle de la crise est écartée par Juglar. Désormais, le cycle est un écart quantitatif procédant aussi bien d'une altération des structures (le développement industriel rapide et les déséquilibres en cours vis-à-vis de l'agriculture) que d'un dérèglement dans l'exercice des fonctions (le crédit et les paiements). Mais le cycle est aussi, en quelque sorte, le risque du développement économique et c'est en ce sens que, sur le plan social, chez Juglar, est pensée de façon novatrice l'articulation entre le normal et le pathologique. Juglar situe sa vision du cycle économique dans le cadre d'une conception où les maladies, pour reprendre ici les termes qu'utilise aujourd'hui G. Canguilhem pour qualifier le vivant, « sont des crises de la croissance vers la forme et la structure adultes des organes, de la maturation des fonctions d'autoconservation interne et d'adaptation aux sollicitations externes $»^{44}$.

Cette idée de la normalité du cycle économique chez Juglar est en fait solidaire de l'ethos bourgeois et réformiste de son temps ${ }^{45}$. Il n'est pas le seul, loin de là, en France, à articuler le thème du progrès, lent et mesuré, et celui du cycle. $P$. J. Proudhon, par exemple, à la même date, fait du balancement des contraires la condition même du mouvement et de la paix. L'idée de cycle charrie de fait des considérations morales et politiques en phase avec la société bourgeoise des années 1850, société se fiant à la propriété, à la famille et à la capacité : le progrès s'inscrit dans la durée et la participation à ses fruits paraît, via le cycle, accessible à tous. Le cycle est la garantie de la fluidité sociale et d'une société « industrielle » où classes et antagonismes sociaux n'ont plus (ou plus que peu) de réalité. C'est ce qu'exprime parfaitement un texte du Journal des débats, en 1847 :

Il n'y a pas de classes en France dans le sens rigoureux du mot. La bourgeoisie n'est pas une classe, c'est une position ; on acquiert cette position, on la perd. Le travail, l'économie, la capacité la donnent ; le vice, la dissipation, l'oisiveté la font perdre. La bourgeoisie est si peu une classe que les portes en sont ouvertes à tous le monde pour en sortir comme pour y entrer ${ }^{46}$.

La lecture des autres textes que publie Juglar dans les années 1850 signale la présence des mêmes thèmes. Les phénomènes démographiques révèlent eux-aussi la présence d'un cycle régulateur - «une soupape de sécurité employée par la Providence pour modérer la marche croissante des peuple» - dans une société en progrès, notamment sur le plan technologique ${ }^{47}$; les pratiques "guerrières " reculent inexorablement devant la raison « industrielle ", ce que signale l'échec de la colonisation en Algérie ${ }^{48}$. Enfin un équilibre dynamique s'établit naturellement entre agriculture et industrie. L'industrie progresse relativement, mais à un rythme sage et

\footnotetext{
${ }^{44}$ G. Canguilhem, Ecrits sur la médecine, Paris, Seuil, 2001, p. 46.

${ }^{45}$ Sur cette question, A. Daumard, Les bourgeois et la bourgeoisie en France depuis 1815, Paris, Aubier, 1987 ; H. G. Haupt, Histoire sociale de la France depuis 1789, Paris, éditions de la MSH, 1993 (ch.2).

${ }^{46}$ Cité dans A. Daumard, Les bourgeois et la bourgeoisie en France depuis 1815, ouv. cit., p. 129.

${ }^{47} \mathrm{Cl}$. Juglar, « De la population en France de 1772 à nos jours (1849) », $1^{\text {er }}$ article, Journal des économistes, vol. 30, sept.-déc. 1851 , p. 378-379.

${ }^{48}$ Cl. Juglar, " De la colonisation en Algérie », $4^{\mathrm{e}}$ article, Journal des économistes, vol. 36, mai-août 1853, p. 232.
} 
lent, que peut seul troubler l'Etat lorsqu'il intervient malencontreusement sur les tarifs douaniers ${ }^{49}$.

\section{Contrôle du cycle économique et scepticisme thérapeutique}

Sur le plan prescriptif, le programme de Juglar, tel qu'il l'expose autour de 1860, est extrêmement timide. Il est patent, explique-t-il, que le cycle affecte durement les «conditions d'existence de la classe ouvrière »(1857: 38-39). De même que l'entrepreneur a innové et risqué en phase ascendante, il est obligé lors de la phase de dépression de réduire sa production :

La production, lancée sur une grande échelle, est obligée de se ralentir, de se modérer, de réduire les salaires, ou même de renvoyer une partie des ouvriers qu'elle occupe, éveillant chez eux ces sentiments de haine qui se manifestent avec tant de violence dans les révolutions, que ce mécontentement, ce malaise général amènent (1857:39)

Mais c'est, en quelque sorte, le coût social de la croissance et de l'innovation et une connaissance objective du phénomène moderne des crises périodiques, traduite et transmise aux ouvriers, doit constituer un facteur de paix sociale. L'étude scientifique - car basée sur «ce que les chiffres offrent d'eux-mêmes » - de cette quasi « loi économique » (1857:35) mérite, en effet, d'être réalisée, « pas tant pour indiquer un remède qui se trouve dans la nature des choses, que pour mesurer froidement les conséquences, conservant une assez ferme confiance dans l'avenir pour traverser le présent sans se laisser aller à une incertitude fâcheuse » (1857:36). Comme l'explique encore plus clairement Juglar dans un texte légèrement postérieur :

Du mélange de tous les intérêts qui se heurtent et se soutiennent dans la société, résulte un effort commun qui vous dirige et vous pousse vers un but fatal que l'on ne peut éviter. Le développement régulier, continu du commerce et de la richesse des nations n'a pas lieu sans douleurs, sans résistances ${ }^{50}$.

Juglar a pu ici puiser son scepticisme à plusieurs sources distinctes ; d'abord, et avant tout sans doute, à la doctrine de l'école libérale française. Novatrice et dynamique au début des années 1840 cette école a entamé un virage nettement conservateur après $1848^{51}$. Elle ne ménage ses critiques ni aux socialistes et républicains, ni aux dirigistes et interventionnistes qui tiennent le gouvernement

\footnotetext{
${ }^{49} \mathrm{Cl}$. Juglar, «Comparaison de la part prise par les produits de l'industrie dans l'augmentation totale du commerce spécial de la France depuis 1829 », Journal des économistes, vol. 37, sept.-déc. 1853.

50 C. Juglar, «Des emprunts et des embarras financiers de la Restauration (1814-1818)», Journal des économistes, 1861, p. 252.

${ }^{51}$ F. Démier, «Les économistes libéraux et 1848 », in P. Dockès et alii. (dir.), Les traditions économiques françaises 1848-1939, Paris, Ed. du CNRS, 2000. L. Levan-Lemesle, Le juste et le riche : l'enseignement de l'économie politique en France, Paris, 2004. Y. Breton, "Les économistes français et le pouvoir politique », Histoire, économie, sociétés, 1982.
} 
aussi bien sous la $2^{\mathrm{e}}$ République qu'au début du Second Empire ; et, avant que Michel Chevalier rallie les libéraux au pragmatisme de l'Empire (vers 1860), le grand auteur libéral au tournant des années 1850 sera Frédéric Bastiat défenseur acharné du libéralisme économique le plus radical. Le scepticisme de Juglar procède également indéniablement de sa foi religieuse et de son enracinement dans un catholicisme qui, autour de 1850 appréhende en France très difficilement la question sociale ${ }^{52}$. Un témoignage inédit, probablement de son épouse, nous apprend que son abandon de la médecine, vers 1848, venait de sa désillusion à guérir le mal :

C'est alors qu'il sentit l'impuissance de la médecine, et qu'à côté du diagnostic il ne trouvait pas de remède efficace et voir toujours souffrir, soulager rarement, compter les jours de chacun sans pouvoir les prolonger, et regardant Dieu comme le seul qui peut guérir il abandonna la médecine pour laisser à son esprit une liberté plus grande et un champs plus vaste ${ }^{53}$.

Ce témoignage nous apprend encore que «ses études de médecine lui avaient appris que les maladies se guérissent difficilement mais se préviennent facilement ». Il est, là encore indéniable que les conception de l'école anatomo-clinique parisienne ont joué un grand rôle dans cette orientation de sa pensée : «le trait dominant et le plus permanent de l'attitude de l'Ecole de Paris en matière de thérapeutique, a pu noter judicieusement E. Ackerknecht, son dénominateur commun en quelque sorte, c'est son scepticisme... Reposant sur la croyance au pouvoir curatif de la Nature, il tend vers l'empirisme $»^{54}$. A partir de 1850, la médecine hospitalière va être en retrait, dépassée par la nouvelle médecine expérimentale développée par une nouvelle génération et s'appuyant désormais sur les approches du laboratoire (physiologie expérimentale, chimie, microscopie). Les cliniciens parisiens, empiristes et praticiens, bloquaient eux sur l'explication des causes des maladies que ni l'examen physique, ni les statistiques, ni l'anatomie pathologique macroscopique ne permettaient finalement de découvrir. Leur attitude avait eu pour résultat positif d'écarter toutes les médications approximatives préconisées avant 1800. Le résultat négatif fut ce grand scepticisme sur le plan thérapeutique. Face à la maladie ces cliniciens faisaient confiance à la médecine expectante et au pouvoir curatif de la nature. Achevant son article «maladie» pour le Dictionnaire de médecine usuelle de J. P. Beaude, A. Hardy, médecin des hôpitaux de Paris plaçait significativement sa confiance en « une réaction salutaire de l'économie opposée à la maladie et en lutte contre ses mauvais résultats. Cette puissance qu'on désigne sous le nom de force médicatrice de la nature, est, comme l'antidote, à côté du poison ; elle tend sans cesse à rétablir le calme et l'équilibre dans l'organisation ${ }^{55}$. Et A. Trousseau, dont Juglar avait suivi le grand cours de thérapeutique à la faculté de médecine, mentionne dans sa Clinique médicale les « actes puissants » de la «nature médicatrice », résumant ensuite la position de l'Ecole parisienne en notant : «Il y a bien longtemps que je suis incliné à croire à l'impuissance de la médecine dans le traitement de la pneumonie aiguë. Il y a bien longtemps que je suis

\footnotetext{
${ }^{52}$ A. Pons, « Catholicisme » in M. Ambrière (dir.), Dictionnaire du 19e siècle européen, Paris, Puf, 1997.

${ }^{53}$ Lettre conservée aux archives familiales.

${ }^{54}$ E. Acknerknecht, La médecine hospitalière à Paris, ouv. cit., p. 176.

${ }^{55}$ Beaude, Dictionnaire usuel de médecine, ouv. cit., vol. 2, p. 389.
} 
tenté de laisser à la nature le soin de mener à bien cette maladie contre laquelle nous sommes tous disposés à agir avec tant de vigueur; mais jusqu'ici je n'ai pas osé le faire $»^{56}$.

\section{Conclusion}

La première édition de Des crises commerciales et de leur retour périodique en France, en Angleterre et aux Etats-Unis (1862) s'achève par deux paragraphes déjà présents dans les textes de 1856 et $1857^{57}$. Ils synthétisent le point de vue de Juglar:

Arrivé au terme de cette étude on ne peut s'empêcher de remarquer la succession régulière des périodes heureuses et malheureuses, traversées par la population française depuis le commencement de ce siècle; tantôt s'élevant à un degré de prospérité inouï pour être précipitée dans les abîmes des révolutions, tantôt sortant de ces abîmes pour atteindre un développement industrielle et un accroissement de richesse inespéré.

Les moyens si simples employés par la Providence pour produire de si grands résultats confondent l'imagination, quand on compare la grandeur des effets à la petitesse des causes.

Une insuffisance de la récolte, augmentant les embarras du commerce et de l'industrie, à la suite de l'exagération et de l'impulsion qui leur avaient été données, détermine une crise souvent suivie d'une révolution, et terminée par une guerre générale ou une grande épidémie. Tout s'arrête pour un temps, le corps social paraît paralysé ; mais ce n'est qu'une torpeur passagère, prélude de plus belles destinées. En un mot, c'est une liquidation générale. Il ne faut donc jamais désespérer ni trop espérer de son pays, se rappelant sans cesse que la plus grande prospérité et la plus grande misère sont sœurs, et se succèdent toujours ${ }^{58}$.

A côté d'autres influences - L'école libérale, les hygiénistes, les catholiques sociaux - se repèrent celles laissées par sa formation en médecine au début des années 1840 à l'apogée de la période «éclectique» de l'Ecole parisienne. En témoignent ses positions sur les chapitres de l'empirisme, de la définition du normal et du pathologique appliqué au monde économique et social, du scepticisme sur le plan prescriptif. C'était probablement la nouvelle définition du normal qui était heuristiquement la plus féconde : elle permettait à Juglar de croiser la thèse libérale du progrès, linéaire car sans fluctuations et seulement contrarié par quelques chocs exogènes, et la thèse socialiste du cycle comme indice des contradictions organiques du capitalisme et donc comme vecteur de son déclin et de son remplacement prochain par un nouveau système économique, politique et social. Juglar, grâce à sa conception du normal impliquant que la mort est intégrée à la vie, croise le thème libéral du progrès et le thème socialiste (et dirigiste) $d u$

\footnotetext{
${ }^{56}$ Cité dans F. Chast, « Les médicaments », in M. Grmek (dir.), Histoire de la pensée médicale en Occident, ouv. cit., vol.2, p. ?

${ }^{57}$ La dernière phrase est originale dans l'édition de 1862.

${ }^{58} \mathrm{Cl}$. Juglar, Des crises commerciales et de leur retour périodique en France, en Angleterre et aux Etats-Unis, ouv. cit., p. 252-253.
} 
cycle : le cycle devient le rythme propre de développement du capitalisme. Cet agencement intellectuel tentait de donner une réponse au blocage du paradigme libéral qui, progressiste et novateur au début des années 1840 (avec notamment des économistes comme Adolphe Blanqui) avait été mis en échec, économiquement et politique entre 1848 et 1851 . Toutefois la proposition de Juglar - ses conceptions vont en outre très peu évoluer par la suite - pouvait difficilement favoriser la réussite d'une nouvelle «situation classique» en économie : sa vision du cycle comme rythme naturel de développement était à la fois trop audacieuse pour des libéraux qui préféraient continuer à miser sur les idées de J.-B. Say en matière de surproduction générale, trop en évident décalage avec les idées socialistes que va rapidement cristalliser la $1^{\mathrm{er}}$ Internationale et trop timide pour les dirigistes et les officiels qui, dès 1856, voyant s'annoncer une nouvelle dépression mirent pragmatiquement en place une première politique anticyclique ${ }^{59}$. Il faudra donc attendre Joseph Schumpeter et les théoriciens modernes du cycle pour que soit véritablement redécouverte l'œuvre de Juglar.

\footnotetext{
${ }^{59}$ Dès mars 1856 une Note officielle parue dans Le Moniteur prévoit une véritable politique anticyclique basée sur le freinage de l'investissement. Sur ce point, L. Girard, La politique des travaux publics sous le Second Empire, Paris, 1952.
} 